\title{
Exploration of College English Classroom Teaching Based on ARCS Motivation Incentive Model
}

\author{
Yuan Zhang \\ Department of English, Busan University of Foreign Studies, Busan, Korea \\ supergirlbecky@aliyun.com
}

Keywords: classroom end link; ARCS motivation incentive model; teaching design.

\begin{abstract}
To effectively motivate students' English learning motivation, based on the ARCS motivation incentive model, the end link of college English classroom was taken as the research object, and the instructional design based on the ARCS motivation incentive model in the end link of college English teaching was explored. The research results showed that the teaching method fully motivated and maintained the motivation of students' learning outside the classroom, and then realized the final learning purpose. It can be seen that improving teachers' awareness focusing on the end link of the classroom, especially based on the motivation and incentive mechanism, linking the teaching opportunities inside and outside the classroom, we can formulate suitable teaching strategies and dig out the great potential usefulness of English teaching.
\end{abstract}

\section{Introduction}

For a long time, English teaching circle in China have made many theoretical discussions and demonstration researches on how to use the motivation to improve English teaching quality. At present, there are many theories about college English classroom and ARCS motivation incentive model, respectively, and made a lot of achievements. Nevertheless, almost all the studies have always stressed that it is the inside classroom completing the task of teaching [1,2], while paid less attention to the link between foreign language classroom and after-school learning. After the teaching content of a class is completed, teachers often say "class's over" or arrange homework to rush over a class [3]. The present researches specifically for the end of class links are relatively small, and the papers on the end of classroom part checked are most researches except for English subject. The studies for English, especially the end of college English classroom, are less. But, specifically for the use of classroom end links, the motive encouragement is conducted to connect the curricular and extra-curricular learning motivation. In addition, studies promoting students' follow-up learning motivation is less.

\section{Background Introduction}

As lifelong learning theory is accepted by more and more people, learning out of physical space classroom has become an indispensable part of people's learning and life [4]. It is not difficult to find if the expansion of the learning environment can be applied being matched with the theory of motivation encouragement, it will make the space of learning English greatly broadened. And based on the review and sorting of the above literature, it can be seen that, many scholars have been fully aware of the importance of the end part of the classroom. Moreover, according to the actual teaching experience and relevant teaching theory, we made valuable studies on the function, principles, and methods and so on of the end link in the course of teaching [5]. From the analysis of the above documents, we find that the types of methods about the end link are various, and the strategies for motivational encouragement are many. However, in how to guide the teaching design of this link, teachers are mostly talking it generally according to the specific experience and teaching theory, while they seldom use the motivation encouragement model of system as the guidance [6]. In addition, researches on the classroom end link of the primary and secondary schools are many, but the studies on the college English class is relatively less [7]. The teaching design for other subjects is many, but it in English is relatively less; in the literature read, the researches design the classroom end link on 
the basis of the motivation incentive model is fragmented and scattered strategies and principle, and it is rare to see the integrated form or theory [8].

\section{Methodology}

This research was carried out in the natural class for teaching implementation by means of convenience sampling. The class was the non-English major students in a college in Fujian in 2014. There were 19 boys and 24 girls, the average age was 18.7 years old, and the average score of the college entrance examination was 98 . The questionnaire was issued two times before and after the implementation of the teaching. 43 copies were issued each time, 43 copies were recovered, and the recovery rate was $100 \%$. A data analysis was made on the 86 questionnaires. The Richter scale in "College Students' Motivation Types and Motivation Intensity" compiled by Gao Yihong (2002) was the deep exploration of Chinese college students' English learning motivation types and motivational intensity; learning motivation and strength scale is the standard measurement tool to understand the foreign language learning motivation under the classroom environment.

This research mainly focuses on the current situation of college English teaching and integrates the main characteristics of the group and the situation of learning specific motivation. It aims at understanding the needs of the relevant students and investigating and analysing the learners' general learning motivation level. Combining with the investigation and analysis of the first stage, on the basis of motivational strategies proposed by John. M. Kelle, the classroom end link is conducted with teaching design. The classroom end link is subdivided into three parts, and the corresponding specific strategies in the ARCS model are performed with encoding according to the division categories. It is fused in the classroom end links and the classroom end link model based on ARCS motivation encouragement model is established. We integrate teaching resources, apply instructional design to specific teaching stages, and adopt the classroom end link model based on ARCS motivation incentive model to practical teaching cases in the end link of class. Subsequently, through questionnaire survey, with the help of the data comparison before and after the implementation of teaching, the motivation level is analysed to evaluate teaching effect and form research results.

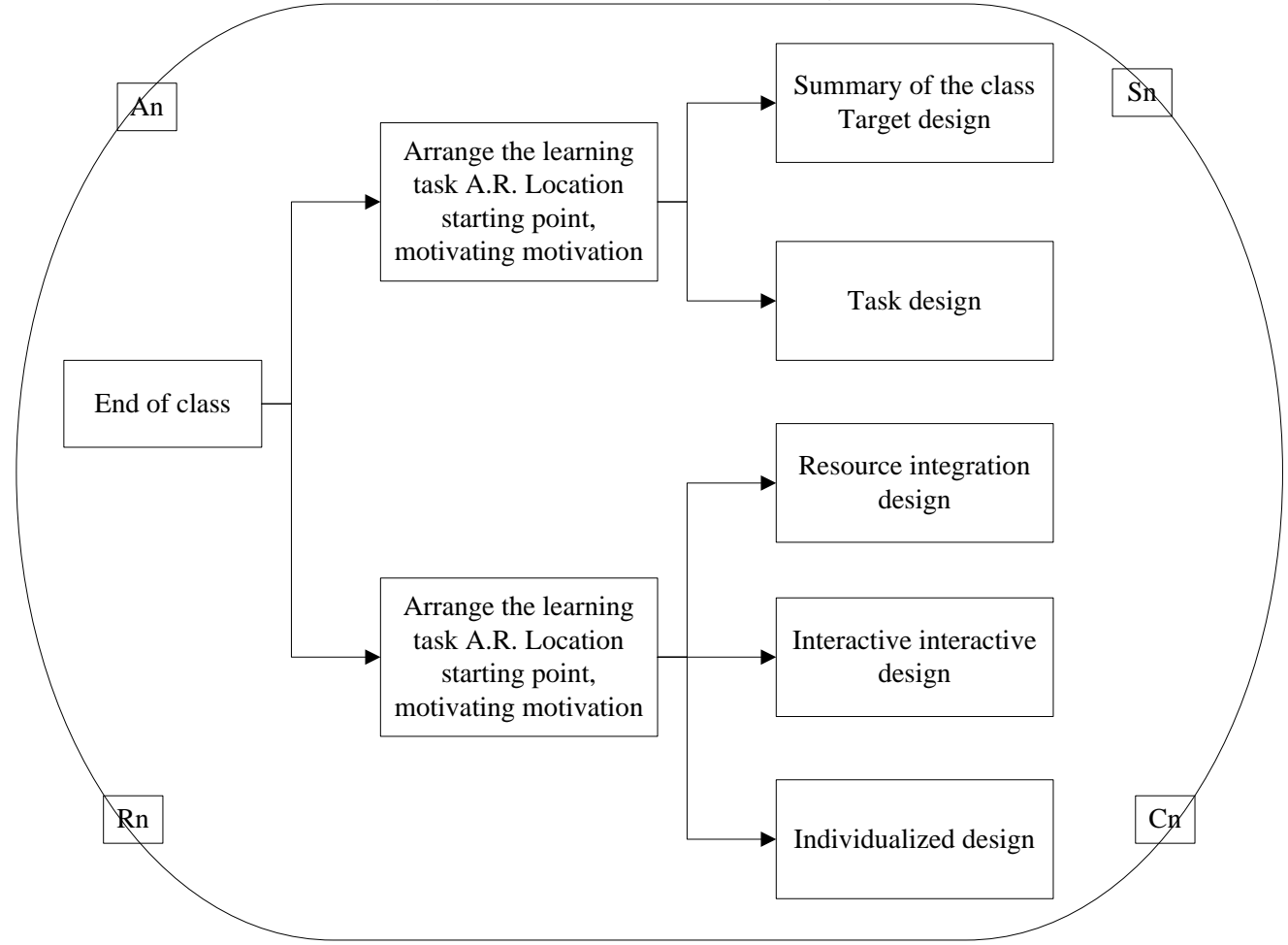

Figure 1. Class end teaching model based on ARCS motivation theory

The classroom end link model we want to build, as shown in Figure 1, is designed from two dimensions, namely, the layout of learning tasks in class and the creation of after-school learning environment. In the design of classroom end link, arousing learners' learning motivation is just the 
first step, but this is not the ultimate goal. The ultimate goal is to make the motivation in and out of the classroom naturally connected and transited and extended to the outside of the classroom to maintain the interest of learners for a long time. As a result, learners continuously find happiness and self-confidence from learning, so that learners not only utilize teaching resources in class, but also make full use of outside resources combined with their own basic characteristics in targeted. From the following three aspects, namely, the determination of goals, the layout of tasks, and the creation of learning environment, the following six aspects are combined with the motivation theory of ARCS to construct the motivation incentive model of the end of college English class link, as shown in Figure 1 and Figure 2.

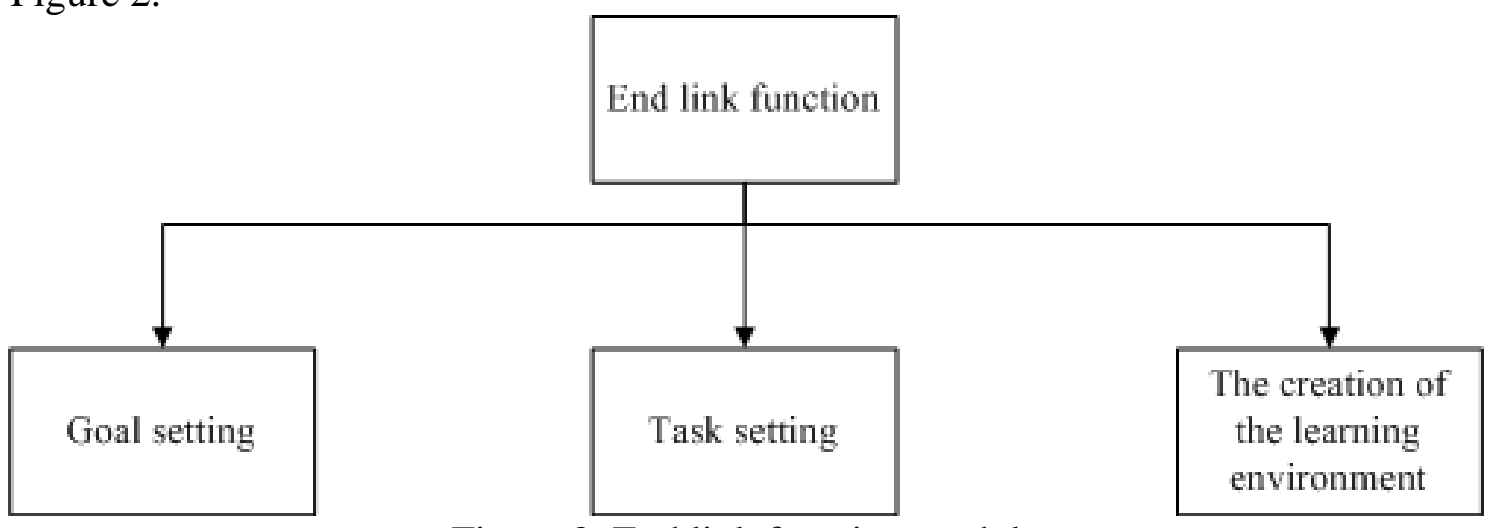

Figure 2. End link function module

\section{Results and Discussion}

\subsection{Design of the Teaching Model of the Classroom End Link Based On ARCS}

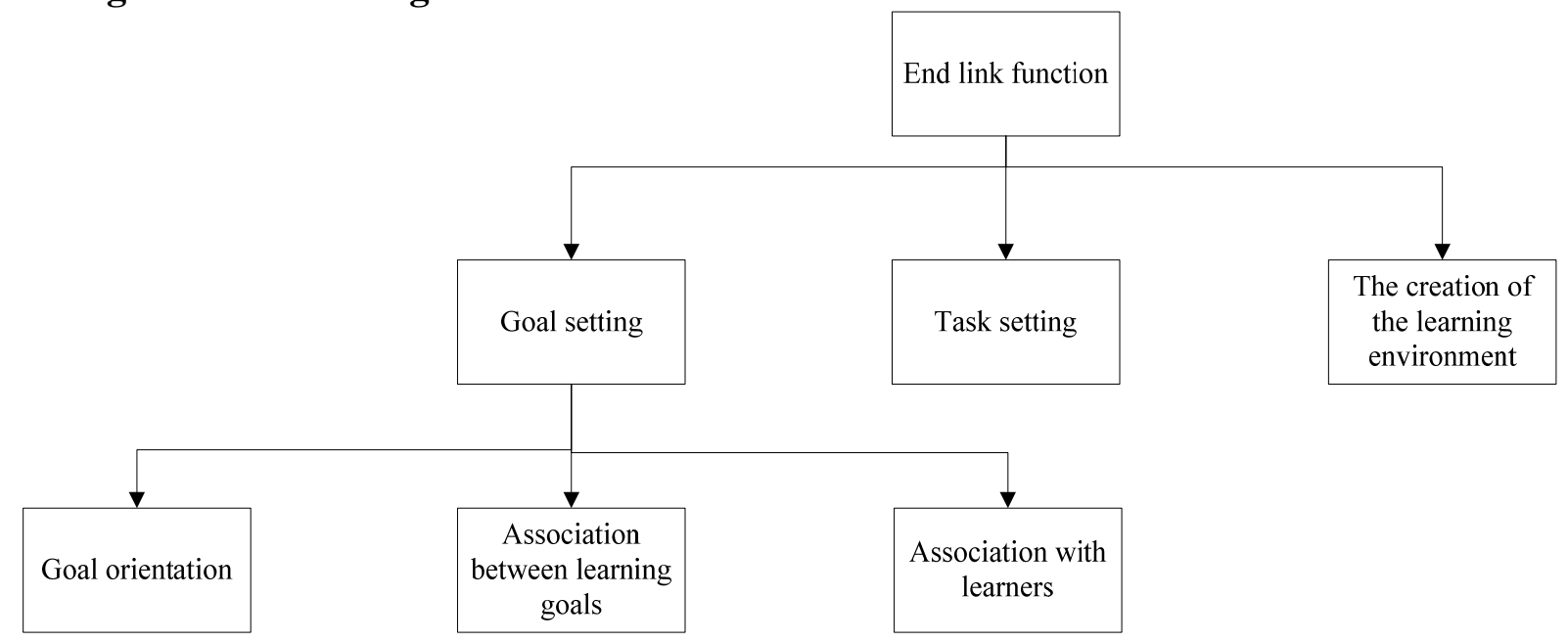

Figure 3. End link target setting module

ARCS theory pointed out that according to a learning task, clear target is beneficial for learners to more efficiently grasp and apply the learned knowledge structure to reasonably estimate their learning goals. If learners can know their learning goals that are expected to fulfil at the beginning of the study, it will be highly beneficial for the establishment of learner's learning confidence. According to the ARCS model, we can know, to enable the learner to systematically study and master the learning content, the most effective motivation incentive and maintaining strategy is to decompose the integrated and systematic learning tasks into several small learning tasks both interrelated and mutually independent, and make learners gradually completing the learning tasks one by one. In this way, learning enthusiasm and confidence of learners will not be destroyed by a huge and complex learning task. When learners focus on the realization of small learning tasks, it not only makes learning easier. More importantly, the learners experience continuously to success in learning, ( $\mathrm{R}$ and $\mathrm{S}$ in ARCS). The learning tasks connected to each other and mutually independent enable learners to learn knowledge together, which help to increase the knowledge transfer rate and bring 
lasting learning motivation for learners. In the ARCS model, the relevance of the learner itself to the target is also a factor that needs to be considered in the class design. Learners' expectations and preparation for their own learning will profoundly affect their learning goals. In turn, learning goals will stimulate learners' learning aspirations and promote learners' learning enthusiasm.

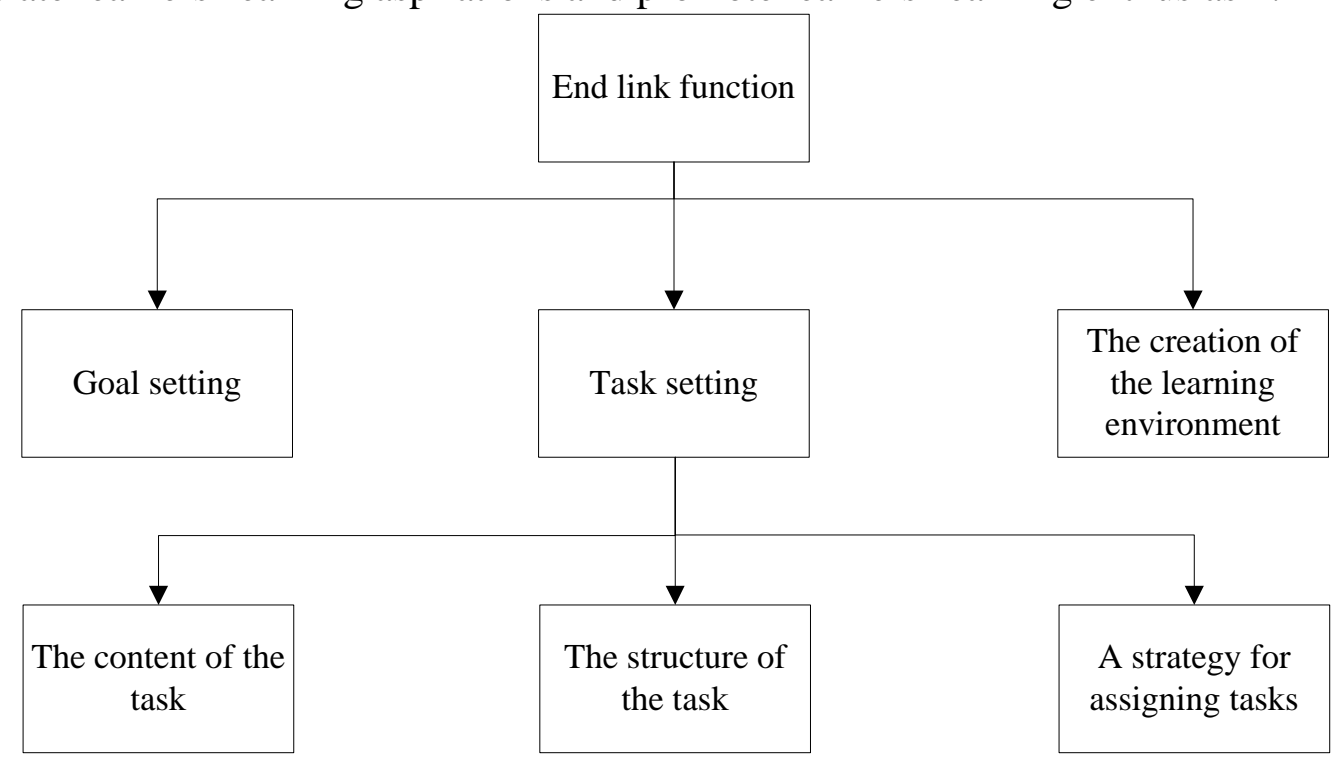

Figure 4. End link task setting module

In the process of college English classroom teaching, the realization of motivation incentive goal should be based on content. The core of the design of classroom termination is the learning task formed according to the selection and organization of learning contents. In the ARCS model, the important way to arouse learners' enthusiasm for learning and keep learning enthusiasm is to protect their scientific knowledge, interactivity, novelty, interest and relevance with learners. In the following, we discuss the content composition design of the classroom end link based on the ARCS model. The content of the task: this part focuses on relevance, that is, design relevant with teaching goals, student value and real life. The structure of the task: this part focuses on the design of confidence and satisfaction. The strategy of assigning tasks: this part is the use of the policy base, and the strategy intervention is carried out when the task is arranged.

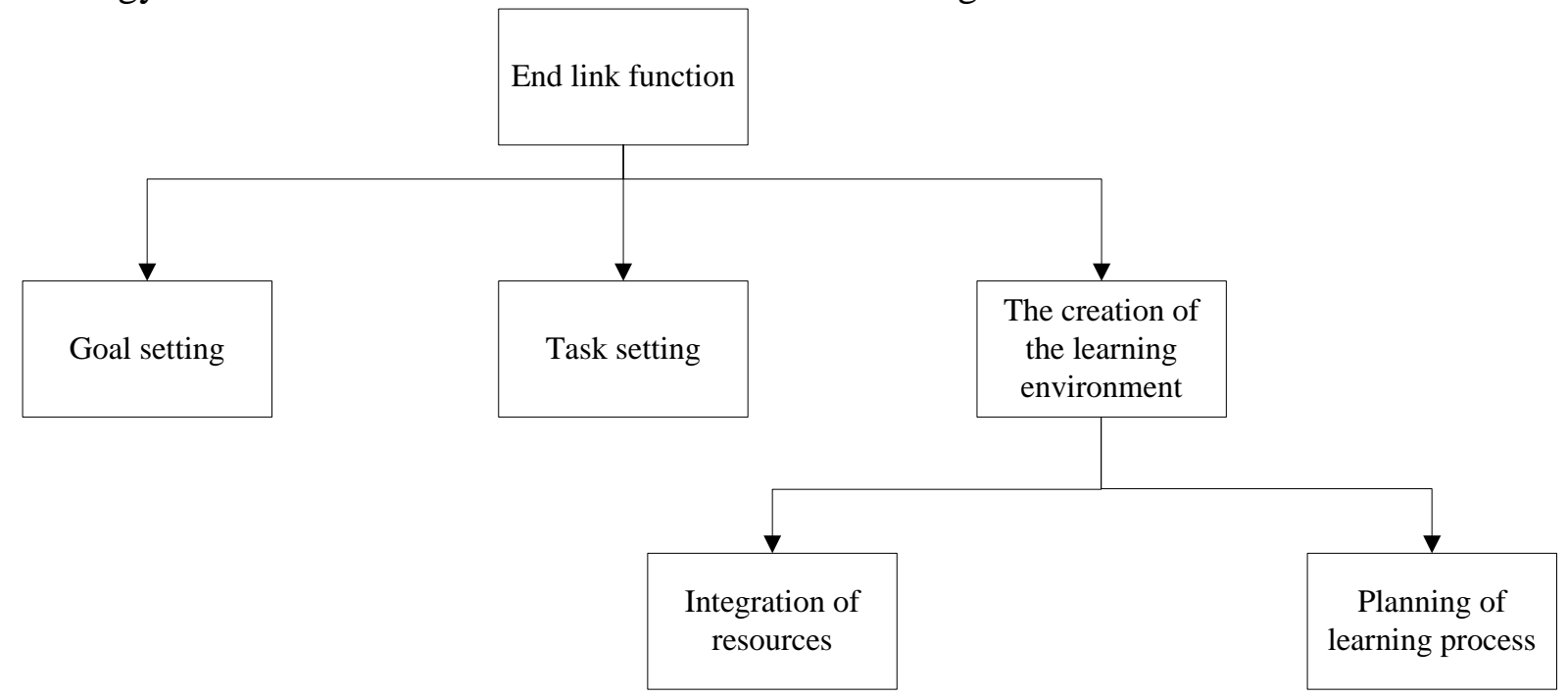

Figure 5. End link learning environment creation module

Learners' attention, learning confidence, learning relevance and learning satisfaction should be emphasized as the four parts, and penetrated into every aspect of learning environment design. The whole learning environment design is fundamentally the process of stimulating and maintaining learning motivation. When the learning tasks are deployed to learners, we should start with the learning enthusiasm of learners and the essence of learning content, to assign appropriate learning tasks for learners. The difficulty of learning tasks stimulates learning ability of learners in a certain 
degree. Learners, in their scope of ability, complete the learning tasks relevant with its force and confidence to achieve their term expectation.

\subsection{Class Case Design Based on ARCS Incentive Model}

In the teaching process, step-by-step teaching mode is applied. According to the normal class process, the teaching content in the teaching arrangement of "New Practical English" is completed. The teaching design is arranged based on the teaching plan in each semester, generally for 6 hours, 3-4 times. Each unit takes the topic as a unit, and it is combined according to easy to complex and step-by-step means. Moreover, in the classroom end link, the ARCS motivation incentive design is carried out.

In this context, in view of the end of the first class of the seventh unit, according to the classroom end link model, the design is shown as follows:

Table 1 . The basic model framework of case design in the classroom end link

\begin{tabular}{|c|c|c|}
\hline \multicolumn{2}{|c|}{ Summing-up on Teaching } & Motivation emphases \\
\hline Task setting & Attention and correlation \\
\hline \multirow{2}{*}{ Learning community creation } & Resource integration & Selective association \\
\cline { 2 - 3 } & Interactive design & Confidence, relevance \\
\cline { 2 - 3 } & Personality display & Confidence, relevance \\
\hline
\end{tabular}

Generally speaking, it is necessary to fully exploit the teaching way in the end, mobilize various resources, and create a rich form. In addition, after entering the preparation stage, we also should pay attention to the design of the environment, help to guide the students wholeheartedly, and create a real scene to help mobilize the enthusiasm of the students. Moreover, in the homework setting, we should also focus on synchronization of the cyber sources. There are several aspects also need special attention: for the group with relatively small size, in the learning, we should give some guidance, summary and so on, and forbid internal imitation of identical group. We should not only focus on the realization of tasks; instead, from the perspective of assessment, the assessment form of group itself is relatively innovative, which cannot be separated from effective supervision. Also, the teaching resources calling should not be too frequent; otherwise, the student may also lose freshness and enthusiasm is not high. In this process, if we can fully mobilize various resources, it will be able to help get better teaching effect to a certain extent, and help strengthening the review results, and in fact, this is also verified.

\subsection{Investigation and Analysis of the Effect of Model Implementation}

After carrying out the teaching implementation of teaching model designed based on ARCS motivation incentive model, all students are investigated, so as to verify the feasibility and effectiveness of the related strategies of the model in the end stage of English teaching.

Table 2. Comparison of motivation types and motivation intensity before and after the implementation of teaching

\begin{tabular}{|c|c|c|c|c|}
\hline & Before implementation & After implementation & Deviation & The value of T \\
\hline Inner interest & $2.0 \pm 0.691$ & $3.07 \pm 0.762$ & 0.97 & 8.25 \\
\hline Achievement motivation & $4.24 \pm 0.485$ & $3.43 \pm 0.618$ & -0.81 & 3.43 \\
\hline Learning situation & $3.10 \pm 0.553$ & $2.68 \pm 0.469$ & -0.42 & 2.17 \\
\hline Go abroad & $1.81 \pm 0.576$ & $2.09 \pm 0.536$ & 0.28 & 1.22 \\
\hline Social responsibility & $3.83 \pm 0.803$ & $3.57 \pm 0.824$ & -0.26 & 1.03 \\
\hline Personal growth & $3.47 \pm 0.716$ & $4.17 \pm 0.497$ & 0.07 & 2.81 \\
\hline Information media & $2.15 \pm 0.691$ & $2.68 \pm 0.632$ & 0.53 & 2.17 \\
\hline Motive strength & $2.07 \pm 0.792$ & $2.92 \pm 0.713$ & 0.85 & 2.98 \\
\hline
\end{tabular}

The same textbooks are used in the natural classes for teaching to verify whether motivation incentive teaching model can improve the motivation of college English learning.

As shown in Table 2, in terms of internal motivation, after teaching implementation, the mean test was 3.07, higher than the pre-test (mean deviation $=0.97$ ), and the value of pre-test and post-test is internal motivation shows obvious difference. The pre-test and post-test results show that the motivation incentive model has an effect on stimulating the students' intrinsic motivation in English at the end of College English. Achievement motivation decreases after teaching implementation, and 
there is a significant difference between the subjects in the pre-test and post-test $(T=3.43)$. This indicates that after the implementation of the teaching model, instrumental motivation can be transformed into intrinsic motivation. In learning contexts, it decreases from 3.10 to 2.68, and after teaching implementation, it drops to 0.42 . This decrease $(T=2.17)$ indicates that the implementation of motivational incentive model reduces situational motivation. The $\mathrm{T}$ value of personal development motivation is 2.81. After the implementation of teaching, students' motivation to go abroad has increased slightly, which increases from the mean of 3.47 in the pre-test to 4.17 in the post-test. For social responsibility motivation, the motivation of the tested is slightly improved, and the mean deviation is 0.26 , but this has no data obvious feature. The $T$ value is 1.03 , indicating that there is no obvious change in motivation before and after the implementation of teaching in terms of the study abroad.

As the motivation of an information medium, the mean value of English increases to 2.92, which increases significantly compared to that before the implementation of the model and this change is meaningful. Data confirm that motivation incentive model helps improve learners' motivation to use English as an information medium. In terms of motivation intensity, the post-test value (mean value is 2.92) is higher than the pre-test value (2.07), and the amplitude is 0.85 ; there is a significant difference $(\mathrm{T}=2.98)$. This shows that the implementation of the teaching model has increased the intensity of students' motivation.

The $\mathrm{T}$ test shows that the seven motives are changed before and after the model is implemented. Among them, the three motives of internal interest, personal development and information communication medium are all strengthened after the experiment. Among them, the motivation of internal interest is the most obvious. At the same time, the motivation of achievement is weakened. The teaching mode makes a specific and clear explanation of learning objectives and learning task $\mathrm{i}$ academic evaluation. The goal and task are natural communication, which does not exceed the students' current English level. Lively and active teaching content and scientific assessment standards stimulate students' interest in learning English and enhance their learning initiative. The interest in learning English is a direct motivational factor in learning a foreign language. Once the learner is interested in learning English and the culture of English, he will have more time and energy to learn English.

Personal development motivation is also changing greatly, as show in (1), before the implementation of teaching. The subjects just start their college life and they have no clear plan for the future. However, this teaching model provides specific goals for learning English. The subjects will spend more time thinking about the future and their personal development.

$$
\operatorname{Dev}=0.70
$$

Learning situation motivation is reduced, as shown in (2), which is a good phenomenon. It reflects that the motivation incentive model makes students no longer rely too much on textbooks and teachers but regards English as an interesting learning project.

$$
\text { Dev }=-0.42
$$

The motivation of English as a medium of information exchange has been strengthened and the deviation is 0.53 . This may be because in the teaching model, the application of modern science and technology makes students realize the important role of English as the medium of obtaining information.

As can be seen from table 2, after the implementation of teaching, motivation is increased and motivation intensity is increased. Under the teaching mode, students spend more time and experience in classroom and extracurricular learning environment. The rich classroom and extracurricular learning activities, as well as the use of intelligent technology in English teaching and learning, promote students to spend more time and experience in learning English. This in turn enhances students' autonomy in learning English. Students unconsciously learn the fun of learning into the relaxed learning environment and after-school time and learn the rich extracurricular activities with fragmentation and frequent contact. It shows that students are actively building English language and discipline knowledge. From the above research, we can find that before and after the implementation of the teaching model, students' motivation level is significantly improved. It indicates that the 
application of ARCS motivation mode in the classroom end link is effective for stimulating the learning motivation of college English.

\section{Conclusion}

This research, based on the college English classroom teaching, applies ARCS motivation incentive model theory in the classroom end links. Combined with the function and structure of the end links, the classroom end links are divided into three modules, namely, the class summary module, after-class task module and learning environment design module. According to ARCS motivation theory, we learn the factors beneficial for college English teaching. Combined with examples, we attempt constructing the strategy base based on ARCS motivation design from the classroom end link. In accordance with the actual situation of college English class and the variation characteristics of students' learning mode, the extrinsic motivation is internalized to stimulate the students' autonomous learning motivation.

After the implementation of the teaching, the proportion of students studying English for pure examination declines. Students spend more time in learning English more actively, so that efforts are made to improve and enhance their motivation intensity. This in turn enhances students' confidence and satisfaction and increases learning autonomy.

\section{References}

[1]. Fu L. based on ARCS model in the design of flipped class teaching. Journal of Inner Mongolia University of Finance and Economics, 2015, 1(6), pp.124-126.

[2]. Guo F, Course introduction and closing strategy. Beijing: Beijing Normal University press, 2013, 2(1), pp. 406-425.

[3]. Meng D. The exploration and practice of motivation stereoscopic English teaching mode. Foreign language audio-visual teaching, 2015, 1(2), pp.50-56.

[4]. Yin L. ARCS motivation incentive model in the application of foreign trade English Teaching in higher vocational colleges. Master's thesis, Shanghai Normal University, 2013, 1(1), pp.781-791.

[5]. Wang L. Research on the function and organizational strategy of the end of the kindergarten collective teaching activities. Master's thesis, Northeast Normal University, 2013, 38(2), pp.414-420.

[6]. Yu X. ARCS motivation design model in high school English teaching design process. Master's thesis, Fujian Normal University, 2013, 23(21), pp.119-128.

[7]. Yu Q, Wang M. The new progress of ARCS research on motivational design model and its application in foreign language teaching. Modern education technology, 2015, 23(19), pp.103-109.

[8]. Yan L, Fan W, Liu Q, et al. Teaching video analysis of network open course based on ARCS motivation incentive model. The success of happiness class and Its Enlightenment from the perspective of learning motivation. Modern education technology, 2013, 1(2), pp.72-75. 